\title{
Intravenous versus intracoronary bolus of glycoprotein IIb/IIIa inhibitor administration during primary percutaneous coronary intervention on long-term left ventricular systolic and diastolic function
}

Pierpaolo Pellicori ${ }^{1,2}$, Concetta Torromeo ${ }^{2}$, Francesco Barillà ${ }^{2}$, Enrico Mangieri $^{2}$ Antonietta Evangelista ${ }^{2}$, Giovanni Truscelli ${ }^{2}$, Pierluigi Costanzo ${ }^{1}$, Angela Hoye ${ }^{1}$, Kenneth Wong ${ }^{1}$

${ }^{1}$ Department of Cardiology, Hull and East Yorkshire Medical Research and Teaching Centre, MRTDS (Daisy) Building, Castle Hill Hospital, Cottingham, UK

${ }^{2}$ Heart and Great Vessel Department, Policlinico Umberto I, Sapienza University, Rome, Italy

\begin{abstract}
Background: In primary percutaneous coronary intervention (PCI), glycoprotein (GP) IIb/IIIa inhibitors are often given in order to attain and maintain better myocardial perfusion. We tested the hypothesis that intracoronary (IC) bolus of GP IIb/IIIa inhibitors might produce a greater improvement in left ventricular $(L V)$ systolic and diastolic function than an intravenous (IV) bolus.

Methods and results: Seventy seven patients undergoing primary PCI for their first ST elevation myocardial infarction (STEMI) were randomly assigned to either an IC or IV bolus of GP IIb/IIIa inhibitor, followed by IV infusion. Compared with the echocardiographic findings within 3 days after PCI, LV ejection fraction was higher at 1 year, with no significant differences between the IV and IC groups (IV: 44\% vs. 49\%, $p=0.001$; IC: $43 \%$ vs. $48 \%$, $p<0.001)$. LV diastolic function $\left(E / E^{\prime}\right)$ did not significantly change at 1 year by either approach.

Conclusions: LV systolic function improved by a similar magnitude following primary PCI, with either IC or IV bolus administration of GP IIb/IIIa inhibitor therapy. However, no significant changes were observed in LV diastolic function. (Cardiol J 2013; 20, 3: 310-317)
\end{abstract}

Key words: ST elevation myocardial infarction, primary percutaneous coronary intervention, intracoronary GP IIb/IIIa inhibitors, diastolic function

\section{Introduction}

Primary percutaneous coronary intervention (PCI) is the preferred reperfusion strategy for patients who suffered an ST-segment elevation myocardial infarction (STEMI) [1]. Despite optimal coronary artery recanalization, distal embolization of atherothrombotic material has important clinical consequences. Over the past decade, numerous randomized placebo-controlled clinical trials have documented the safety and clinical efficacy of intravenous platelet glycoprotein (GP) IIb/IIIa in-

Address for correspondence: Dr Pierpaolo Pellicori, Department of Cardiology, Hull and East Yorkshire Medical Research and Teaching Centre, MRTDS (Daisy) Building, Castle Hill Hospital, Cottingham, Kingston upon Hull, HU16 5JQ, UK, tel: +44 (0)1482-461813, e-mail: pierpaolo.pellicori@hey.nhs.uk 
hibitors in various clinical settings, including some patients with high risk acute coronary syndromes and many patients who undergo primary PCI for acute MI (AMI). GP IIb/IIIa inhibitors have been shown to improve microvascular reperfusion and, consequently, clinical outcomes including reduced re-infarction rate [2-5]. Intracoronary (IC) administration of GP IIb/IIIa inhibitor may increase local drug compared to intravenous (IV) treatment. It may improve clinical outcomes in patients with STEMI undergoing primary PCI. This alternative approach has been investigated in several studies to further characterize the clinical benefits of this mode of drug delivery, but the results are still controversial [6-10]. Ischemic injury after MI affects not only systolic but also diastolic function of the left ventricle (LV). Diastolic dysfunction is associated with and may cause progressive LV dilation. Futhermore, mortality is about 4 times higher in patients with severe diastolic dysfunction after AMI $[11,12]$. The effects of IC administration of GP IIb/IIIa inhibitors on diastolic function have not been studied. Therefore, we tested the hypothesis that IC bolus administration of a GP IIb/IIIa inhibitor exerts a superior effect on both LV systolic and diastolic function compared to an IV bolus.

\section{Methods}

\section{Patients}

Between March 2007 and December 2008, 77 patients admitted into the Department of Heart and Great Vessels, Sapienza University, Rome, with their first STEMI undergoing primary PCI were randomly assigned in a 1:1 ratio to receive either IC or IV bolus GP IIb/IIIa inhibitor therapy followed by continuous infusion. Inclusion criteria were the presence of MI symptoms for less than 12 hours in duration and ST elevation of at least $0.1 \mathrm{mV}$ in more than 2 limb leads or at least $0.2 \mathrm{mV}$ in 2 or more contiguous precordial leads. The study conforms to the principles outlined in the Declaration of Helsinki and was approved by our local research ethics committee. All patients gave written informed consent.

\section{Intravenous GP IIb/IIIa inhibitor group}

The IV bolus GP IIb/IIIa inhibitor (abciximab: $0.25 \mathrm{mg} / \mathrm{kg}$ body weight or eptifabitide: $180 \mu \mathrm{g} / \mathrm{kg}$ body weight) was given after stent deployment, through the arterial sheath, and was followed by a 12 -hour abciximab $(0.125 \mu \mathrm{g} / \mathrm{kg} / \mathrm{min})$ or 24 -hour eptifibatide $(2.0 \mu \mathrm{g} / \mathrm{kg} / \mathrm{min})$ IV infusion, given their different pharmacokinetic variables which might influence their effect [13]. The decision to give eptifibatide or abciximab was taken at the discretion of the interventional cardiologist.

\section{Intracoronary GP IIb/IIIa inhibitor group}

Abciximab or eptifabitide was given as single bolus after infarct related artery recanalization, when the stent was deployed, directly through the PCI guide catheter, followed by 12 -hour abciximab $(0.125 \mu \mathrm{g} / \mathrm{kg} / \mathrm{min})$ or 24 -hour eptifibatide $(2.0 \mu \mathrm{g} /$ $/ \mathrm{kg} / \mathrm{min}$ ) IV infusion.

\section{Details of PCI and other adjunctive therapies}

All stent deployments were preceded by balloon predilatation. All patients received $300 \mathrm{mg}$ aspirin and heparin (70 U/kg) IV before PCI and $600 \mathrm{mg}$ clopidogrel loading dose afterwards, followed by $75 \mathrm{mg}$ daily for 12 months. All patients had similar treatment for secondary prevention, which was started in a coronary care unit, and then up-titrated accordingly during the follow-up period. All patients were still taking clopidogrel $75 \mathrm{mg}$ when they had their 12-month follow-up assessment. Clopidogrel was not administered earlier in case if patients had 3 vessel coronary artery disease or left main disease requiring coronary artery bypass graft surgery. All patients received low molecular heparin for 48 hours after the procedure.

\section{Echocardiography}

All patients underwent an echocardiogram within 3 days after their primary PCI. An echocardiogram at 12 months was possible for only 65 patients. Two patients died (1 from each group), 8 preferred to be followed up in another department and 2 lived in a different region. Echocardiographic results presented were analyzed by 1 single observer (PP) blinded to the route of administration of GP IIb/IIIa inhibitor therapy. The study was carried out with Toshiba Aplio CV. Left ventricular end diastolic (LVEDV) and end systolic volumes (LVESV) were obtained from the apical 2- and 4-chamber views, according to the Biplane Simpson's method. Left ventricular ejection fraction (LVEF) was defined as (LVEDV-LVESV)/LVEDV $\times 100 \%$. Pulsed Doppler examination of the LV inflow was performed with the sample volume placed between the tips of the mitral valve leaflets and early diastolic (E) wave, the atrial (A) wave, E/A ratio, and the $\mathrm{E}$ deceleration time were measured. Pulsed tissue Doppler imaging (TDI) was used to record the velocity profile at the septal and lateral mitral annulus and the peak systolic (S'), early diastolic (E') and atrial (A') velocity were recorded. The ratio 
Table 1. Patients characteristics.

\begin{tabular}{|c|c|c|c|}
\hline Characteristics & $\begin{array}{l}\text { Intracoronary bolus } \\
\qquad(\mathrm{n}=38)\end{array}$ & $\begin{array}{l}\text { Intravenous bolus } \\
\text { ( } n=39)\end{array}$ & $\mathbf{P}$ \\
\hline Age [range] & $59(39-77)$ & $59(41-82)$ & 0.94 \\
\hline Male & $34(87 \%)$ & $29(76 \%)$ & 0.21 \\
\hline \multicolumn{4}{|l|}{ Cardiovascular risk factors: } \\
\hline Hypertension & $19(48 \%)$ & $20(51 \%)$ & 0.91 \\
\hline Smoker & $20(52 \%)$ & $24(61 \%)$ & 0.43 \\
\hline Hypercholesterolemia & $27(71 \%)$ & $28(71 \%)$ & 0.94 \\
\hline Diabetes mellitus & $9(23 \%)$ & $8(20 \%)$ & 0.73 \\
\hline Anterior myocardial infarction & $22(58 \%)$ & $22(56 \%)$ & 0.71 \\
\hline Cardiogenic shock & $6(15 \%)$ & $3(8 \%)$ & 0.26 \\
\hline Peak troponin [ng/mL] & $90.6 \pm 79.5$ & $89.7 \pm 75.9$ & 0.95 \\
\hline Coronaries $>70 \%$ stenosis & $1.7 \pm 0.8$ & $1.6 \pm 0.8$ & 0.33 \\
\hline \multicolumn{4}{|l|}{ GP IIb/Illa inhibitor: } \\
\hline Abciximab & $17(45 \%)$ & $18(46 \%)$ & 0.89 \\
\hline Eptifabitide & $21(55 \%)$ & $21(54 \%)$ & 0.92 \\
\hline
\end{tabular}

Patients characteristics: Continuous variables are showed as mean (standard deviation). Categorical variables are expressed as count (\%).

between peak $\mathrm{E}$ and $\mathrm{E}$ ' recorded at lateral mitral annulus was calculated to estimate $\mathrm{LV}$ filling pressures (E/E' lat). Three left atrial (LA) dimensions were obtained to calculate the LA volume. The first (SA1) was measured by 2-dimensional guided M-mode echocardiography obtained in the parasternal short axis view at the base of the heart, the second (SA2) and the third (SA3) were obtained measuring the short and the long axis dimensions in the apical 4-chamber view at ventricular endsystole. Volume was calculated by the formula $\pi / 6$ $(\mathrm{SA} 1 \times \mathrm{SA} 2 \times \mathrm{SA} 3)$. All the parameters obtained with pulsed wave Doppler and TDI were measured from 3 cardiac cycles and then averaged. The EF calculations were performed from 1 heart beat.

\section{Statistical analysis}

The effects of each route of administration on LV volumes, systolic and diastolic function were assessed by comparing the echocardiographic findings within 3 days after PCI with data obtained at 1 year follow-up. The effects of the 2 routes of administration of GP IIb/IIIa inhibitors (IV vs. IC) on LV volumes, systolic and diastolic function were compared using independent and paired samples t-test for continuous variables. Proportions were assessed using $\chi^{2}$ test. All variables were normally distributed. Analyses were performed using SPSS software, a p-value $<0.05$ was considered statistically significant.

\section{Results}

\section{Clinical characteristics}

The study included 77 patients with a median age of 59 (range 39-82) years; 38 were assigned to IC and 39 to IV GP IIb/IIIa bolus administration. The baseline characteristics are shown in Table 1 and were similar in both groups. No differences were found between the two groups in terms of sex, age, cardiovascular risk factors, number of coronary arteries with significant stenosis, presence of anterior MI and peak troponin release.

Effects of different routes of administration of GP IIb/IIIa inhibitors on left ventricular function and remodeling

Overall, echocardiographic LV size, systolic and diastolic function in the immediate post-infarction period were similar for the 2 routes of administration (Table 2).

Compared with the immediate post-infarction evaluation, LVEF was higher at 1 year, with no significant differences between groups (IV: $44 \%$ vs. $49 \%, \mathrm{p}=0.001$; IC: $43 \%$ vs. $48 \%, \mathrm{p}<0.001)$. Patients who received IC GP IIb/IIIa inhibition had a significant increase in LVEDV (from $120 \mathrm{~mL}$ 3 days after primary PCI to $139 \mathrm{~mL}$ at 1 year, $\mathrm{p}=$ $=0.001)$. By way of contrast, there was no significant increase in the LVEDV in the cohort who received the IV GP IIb/IIIa inhibitor bolus. Nev- 
Table 2. Echocardiographic measurements obtained during conventional transthoracic echocardiography in patients who received intracoronary (IC) or intravenous (IV) bolus of GP Ilb/Illa inhibitors (within 3 days from primary percutaneous coronary intervention $[\mathrm{PCl}]$ and at 1 year follow up).

\begin{tabular}{|c|c|c|c|c|c|c|}
\hline & \multicolumn{3}{|c|}{3 days after primary $\mathrm{PCI}$} & \multicolumn{3}{|c|}{1 year after primary $\mathrm{PCI}$} \\
\hline & $\begin{array}{l}\text { IC bolus } \\
(n=38)\end{array}$ & $\begin{array}{l}\text { IV bolus } \\
(n=39)\end{array}$ & $\mathbf{P}$ & $\begin{array}{l}\text { IC bolus } \\
(n=31)\end{array}$ & $\begin{array}{l}\text { IV bolus } \\
(n=34)\end{array}$ & $\mathbf{P}$ \\
\hline LVEDV [mL] & $120 \pm 34$ & $121 \pm 30$ & 0.78 & $139 \pm 39$ & $133 \pm 40$ & 0.59 \\
\hline LVESV [mL] & $65 \pm 31$ & $68 \pm 23$ & 0.71 & $73 \pm 34$ & $70 \pm 32$ & 0.70 \\
\hline LVEF [\%] & $43.1 \pm 9.2$ & $44.1 \pm 7.9$ & 0.28 & $48.5 \pm 10.7$ & $48.7 \pm 8.5$ & 0.94 \\
\hline E DT [ms] & $191 \pm 39$ & $204 \pm 57$ & 0.33 & $227 \pm 56$ & $217 \pm 60$ & 0.51 \\
\hline E/A ratio & $0.98 \pm 0.26$ & $1.11 \pm 0.47$ & 0.12 & $1.03 \pm 0.59$ & $1.03 \pm 0.53$ & 0.97 \\
\hline TDI S: Septal [cm/s] & $6.86 \pm 1.03$ & $6.96 \pm 1.18$ & 0.67 & $7.09 \pm 1.59$ & $7.61 \pm 1.45$ & 0.17 \\
\hline TDI E: Septal [cm/s] & $6.24 \pm 1.67$ & $7.45 \pm 2.44$ & 0.01 & $6.89 \pm 2.08$ & $7.22 \pm 1.93$ & 0.50 \\
\hline TDI A: Septal [cm/s] & $8.68 \pm 1.77$ & $8.66 \pm 1.82$ & 0.93 & $8.95 \pm 2.46$ & $9.53 \pm 2.23$ & 0.32 \\
\hline TDI S: Lateral [cm/s] & $7.23 \pm 1.16$ & $7.32 \pm 1.22$ & 0.93 & $8.01 \pm 1.97$ & $8.15 \pm 1.89$ & 0.76 \\
\hline TDI E: Lateral [cm/s] & $9.25 \pm 2.42$ & $9.49 \pm 2.6$ & 0.67 & $8.79 \pm 2.69$ & $9.63 \pm 2.64$ & 0.21 \\
\hline TDI A: Lateral [cm/s] & $9.03 \pm 1.78$ & $8.82 \pm 2.02$ & 0.64 & $9.55 \pm 3.27$ & $9.45 \pm 3.17$ & 0.89 \\
\hline LA volume [mL] & $54 \pm 20$ & $58 \pm 14$ & 0.28 & $63 \pm 25$ & $63 \pm 16$ & 0.27 \\
\hline $\mathrm{E} / \mathrm{E}^{\prime}$ lateral & $7.68 \pm 2.88$ & $7.92 \pm 3.76$ & 0.79 & $7.81 \pm 3.39$ & $7.57 \pm 2.89$ & 0.24 \\
\hline
\end{tabular}

LVEDV - left ventricular end diastolic volume; LVESV — left ventricular end systolic volume; LVEF — left ventricular ejection fraction; DT — deceleration time; LA — left atrium; TDI — tissue Doppler imaging

ertheless, at 1 year LVEDV were similar for both groups of patients $(139 \pm 39 \mathrm{~mL}$ vs. $133 \pm 40 \mathrm{~mL}$, $\mathrm{p}=0.59)$ and this apparent difference between the 2 routes of administration was also not statistically significant $(19.7 \mathrm{~mL}$ in the IC cohort vs. $12.6 \mathrm{~mL}$ in the IV cohort, $\mathrm{p}=0.33$; Table 3 ).

Also, peak systolic and diastolic velocities recorded with TDI at the septal or lateral mitral annulus were not different between the two groups at 3 days post PCI or at 1 year follow-up (Table 2).

Overall, LV diastolic function did not change between the immediate post-infarction assessment and 1 year with either method of administration. Neither approach altered the patients' E/E', the key parameter for $\mathrm{LV}$ diastolic function evaluation. A statistically significant increase in $\mathrm{E}$ deceleration time was found at 1 year in the group treated with IC GP IIb/IIIa inhibitors (191 ms [at 3 days] vs. $227 \mathrm{~ms}$ [at 1 year], $\mathrm{p}=0.001$ ) and these patients ended up with greater LA volume at 1 year $(63 \mathrm{~mL})$, compared with 3 days post PCI $(54 \mathrm{~mL}, \mathrm{p}=0.018)$. However, LA volume and $\mathrm{E}$ deceleration time did not different between these two groups 3 days after PCI or after 1 year follow-up (Table 2).

\section{Discussion}

LV systolic function improved by a similar extent following primary PCI with both IC and
IV bolus of GP IIb/IIIa inhibitor adjunctive therapy. Neither IV nor IC bolus administration of a GP IIb/IIIa inhibitor appeared to significantly alter LV diastolic function at 1 year.

Platelet GP IIb/IIIa inhibitors block the final pathway of platelet aggregation. Following the publication of several randomized trials [14-16] and 2 meta-analyzes [17, 18], the revised ACC/AHA STEMI guidelines in 2009 [19] focused on the role of IV administration of GP IIb/IIIa inhibitors in this setting. The use of GP IIb/IIIa receptor inhibitors was reviewed by the Task Force who concluded that, in the era of dual-antiplatelet therapy with heparin or bivalirudin, the evidence indicated that adjunctive use of a GP IIb/IIIa antagonist could be useful in selected patients at the time of primary PCI but could not be recommended as routine therapy. This is because treatment with these agents did not result in a statistically significant difference in 30-day mortality or re-infarction or urgent target vessel revascularization. These questions are still debated and 2 recent meta-analyzes [20, $21]$ concluded that, compared to the IV route, IC administration of GP IIb/IIIa had favourable effects in reducing short-term mortality after PCI. However, results from AIDA STEMI, the largest trial assessing clinical outcomes in patients receiving IC abciximab [22], did not demonstrate a favourable effect of IC vs. IV approach. These results have 
Table 3. Analysis of variations in systolic and diastolic echocardiographic measurements in patients treated with intracoronary (IC) or intravenous (IV) bolus of GP Ilb/Illa inhibitors between baseline (within 3 days after percutaneous coronary intervention) and 1 year follow up.

\begin{tabular}{lccc} 
& IC bolus $(\mathbf{n}=\mathbf{3 1})$ & IV bolus $(\mathbf{n}=\mathbf{3 4})$ & $\mathbf{P}$ \\
\hline LVEDV [mL] & $19.67 \pm 30.34$ & $12.58 \pm 28.53$ & 0.33 \\
LVESV [mL] & $8.44 \pm 30.09$ & $2.06 \pm 21.33$ & 0.32 \\
LVEF [\%] & $5.38 \pm 7.16$ & $4.50 \pm 7.01$ & 0.61 \\
E DT [ms] & $35.71 \pm 72.38$ & $12.44 \pm 77.96$ & 0.22 \\
E/A ratio & $0.17 \pm 0.53$ & $-0.05 \pm 0.57$ & 0.61 \\
TDI S: Septal [cm/s] & $-0.56 \pm 2.03$ & $0.45 \pm 1.55$ & 0.10 \\
TDI E: Septal [cm/s] & $0.94 \pm 2.68$ & $-0.27 \pm 2.70$ & 0.16 \\
TDI A: Septal [cm/s] & $-1.03 \pm 3.37$ & $0.48 \pm 1.88$ & 0.08 \\
TDI S: Lateral [cm/s] & $-0.41 \pm 2.92$ & $-0.02 \pm 2.38$ & 0.62 \\
TDI E: Lateral [cm/s] & $-0.31 \pm 2.58$ & $0.60 \pm 2.74$ & 0.31 \\
TDI A: Lateral [cm/s] & $-1.01 \pm 3.1$ & $0.46 \pm 2.91$ & 0.14 \\
LA volume [mL] & $8.57 \pm 18.98$ & $4.41 \pm 15.71$ & 0.34 \\
E/E' lateral & $0.12 \pm 4.01$ & $-0.35 \pm 4.29$ & 0.64 \\
\hline
\end{tabular}

LVEDV — left ventricular end diastolic volume; LVEVS — left ventricular end systolic volume; LVEF — left ventricular ejection fraction; DT — deceleration time; LA — left atrium; TDI — tissue Doppler imaging

been attributed to be due to the fact that patients were at low risk of adverse events and the study ended up with inadequate power in assessing mortality [23]. The same authors added the AIDA results in a meta-analysis of available trials, and concluded that there was no longer any evident superiority of IC over IV abciximab administration in STEMI patients [23]. Interestingly, the AIDA STEMI trial [22] showed that the adjunctive use of an IC bolus may reduce the rate of new onset of heart failure at 3 months after an acute event compared with an IV bolus: this end point might be considered an important target of therapy and may be related to an effect on LV filling pressures. In fact, non-invasive estimation of high LV filling pressures after a MI is one of the most powerful predictors of poor outcome [12], more than other measures of the LV systolic function.

Intracoronary bolus administration of GP IIb/ /IIIa inhibitors may increase local drug levels and it may improve LV function in patients with STEMI undergoing primary PCI. In a seminal paper Neumann et al. [24] demonstrated that the addition of adjunctive IV abciximab therapy resulted in improved global LVEF 2 weeks (but not immediately) after stent placement. This could probably be explained by myocardial stunning which took some time to resolve. Further, in an magnetic resonance imaging (MRI) study, Thiele and colleagues found no significant improvement in LVEF and volumes
1 to 4 days after an acute STEMI [6]. Thus, our first echocardiogram performed within 3 days after a STEMI appears representative of LV function post-MI. Overall, there was no significant difference between the effects of IC and IV administration of GP IIb/IIIa inhibitors on LV systolic function at 1 year of follow-up. A recent paper by Eitel et al. [25] examined 6-month follow-up data of 154 STEMI patients undergoing PCI randomized to either IC or IV abciximab with subsequent 12 hours IV infusion. They found the infarct size as assessed by delayed enhancement MRI was significantly reduced in the IC abciximab group. In keeping with the findings of the present study, as expected, IC abciximab was also found in their study to have improved LVEF. Thus, we can be confidant that IC GP IIb/IIIa inhibitor delivery results in improved LVEF 6 months after MI, and this improvement appears to be maintained at 12 months. However, in contrast to our findings, Eitel et al. [25] reported no improvement in LVEF if patients were given IV abciximab. This observation by Eitel's group is also at odds against the finding reported by Neumann et al. [24] as we discussed earlier.

The recently reported ICE trial [8] showed IC eptifibatide during PCI for ACS resulted in higher local platelet GP IIb/IIIa receptor occupancy, which was associated with better microvascular perfusion demonstrated by an improved corrected TIMI frame count. However, as in our study, a loading 
dose of clopidogrel was administered after completion of PCI. In the larger CICERO trial [26], patients were pre-treated with clopidogrel. The primary end point was the incidence of restored myocardial reperfusion, defined as complete ST-segment resolution. Secondary end points included myocardial reperfusion as assessed by myocardial blush grade, enzymatic infarct size, and major adverse cardiac events at 30 days. The incidence of complete ST-segment resolution was similar in the IC and IV groups. However, the incidence of myocardial blush grade $2 / 3$ was higher in the IC group than in the IV group. Moreover, enzymatic infarct size was smaller in the IC than in the IV group. Nevertheless, disappointingly, the incidence of major adverse cardiac events was similar in both groups (5.5\% vs. $6.1 \%, \mathrm{p}=0.786)$. Eitel et al. [25] also noted that although there was MRI evidence of smaller infarct size in the IC group, this did not translate into improvement in event free survival. Thus, there was no significant difference between the 2 strategies in hard outcome such as death, reinfarction or target vessel revascularization at 6 months.

The IC approach has been investigated in several studies but none of them studied LV diastolic function as a primary endpoint. Our pilot study adds to the existing literature and debate in several important ways. First, we have painstakingly studied LV diastolic function and did not observe any significant difference between the 2 treatment strategies in their ability to alter LV diastolic function at 1 year. LV filling pressures had been examined measuring the flow velocities across the mitral valve during early diastole and during atrial filling (E/A ratio) for decades [27], but it is difficult to distinguish the normal pattern and the "pseudonormal" pattern seen in moderate diastolic dysfunction. The use of TDI and the $\mathrm{E} / \mathrm{E}$ ' ratio has been nowadays widely introduced in clinical practice because it is less load dependent and offers a good estimation of LV filling pressures [28]. Neither IV or IC approach altered the patients' E/E' following primary PCI at 1 year. Second, our longer duration of follow-up of 1 year may have explained some of the differences between our study and that of Eitel et al. [25]. We do not have interim 6 months follow-up data for comparison. However, we found LVEF improved similarly with both IC and IV strategies.

\section{Limitations and ideas for future research}

Our study is small and cannot exclude subtle differences between routes of administration. Fur- ther studies focusing on the improvement of long-term diastolic function are warranted, however, a large effect seems unlikely.

The pain to balloon time was not available for all patients and it has not been considered in our analysis. Nevertheless, the ventricular function was similar 3 days after MI for both groups, which makes it unlikely that one group included more patients who had much longer pain to needle time, which would have led to worse ventricular function. In any case, they all had their primary PCI within 12 hours of pain onset.

We did not assess parameters which could give information about restoration of myocardial perfusion after PCI. However, in the large AIDA STEMI [22], an IC bolus of GP IIb/IIIa inhibitors did not result in a difference in infarct size or improved perfusion as compared with an IV bolus.

Our study allowed the use of eptifibatide or abciximab (at the discretion of the cardiologist). Nevertheless, they appear similarly effective in the primary PCI setting [29].

Thrombus aspiration device was not used prior to balloon angioplasty and stent implantation. As discussed earlier, however, in patients undergoing primary PCI with thrombus aspiration, IC administration improved myocardial reperfusion as assessed by myocardial blush grade and led to a smaller enzymatic infarct size but it did not improve the primary endpoint of that study, i.e. ST-segment resolution.

We did not consider target vessel revascularization and mortality as end point in our study given the small number of patients and the limited number of events expected. However, a recent study has shown that the IC use of GP IIb/IIIa decreased target vessel revascularization [30] despite the fact that its overall rate $(6.5 \%)$ was relatively high, as the authors pointed out themselves. Remarkably, further, the study found an absolute reduction of mortality at 30 days from $5.3 \%$ in the IV group to $1.1 \%$ in the IC group.

There remain several unresolved issues whether there remains benefit in the era of new antiplatelet therapies and also whether there is benefit when used in addition to thrombectomy. Further, we did not consider the incidence of bleeding as secondary end-point. However current evidence suggests no difference in bleeding complications between the two different strategies [21, 31].

\section{Clinical implications}

Current evidence indicates that adjunctive use of a GP IIb/IIIa antagonist can be useful in selected patients and there is a general consensus amongst 
the interventional cardiology research community that IC bolus of GP IIb/IIIa inhibition is (probably) superior to IV in terms of improving outcome (driven by differences at 30 days). However, there is still no study that is adequately powered to look at this properly with respect to a difference in death in particular over a longer period of time.

Even if the IC route has the theoretical advantages of improving GP IIb/IIIa receptor occupancy, as well as improving perfusion and reducing infarct size, in our present pilot study, a different route of administration influenced neither the improvement in LVEF, nor diastolic function at 1 year.

\section{Conclusions}

LV systolic function improved by a similar magnitude with both IC and IV bolus administration of GP IIb/IIIa inhibitor therapy during primary PCI. No substantial differences between the effects of IV or IC bolus administration of a GP IIb/IIIa inhibitor on LV diastolic function were observed during primary PCI for first-time STEMI at 1 year follow-up.

\section{Conflict of interest: none declared}

\section{References}

1. Van de Werf F, Bax J, Betriu A et al. ESC Committee for Practice Guidelines (CPG). Management of acute myocardial infarction in patients presenting with persistent ST-segment elevation. Eur Heart J, 2008; 29: 2909-2945.

2. De Luca G, Suryapranata H, Stone GW et al. Abciximab as adjunctive therapy to reperfusion in acute ST-segment elevation myocardial infarction: A meta-analysis of randomized trials. JAMA, 2005; 293: 1759-1765.

3. Gurm HS, Smith DE, Collins JS et al.; Blue Cross Blue Shield of Michigan Cardiovascular Consortium (BMC2). The relative safety and efficacy of abciximab and eptifibatide in patients undergoing primary percutaneous coronary intervention: Insights from a large regional registry of contemporary percutaneous coronary intervention. J Am Coll Cardiol, 2008; 51: 529-535.

4. Rakowski T, Siudak Z, Dziewierz A et al. Early abciximab administration before transfer for primary percutaneous coronary interventions for ST-elevation myocardial infarction reduces 1-year mortality in patients with high-risk profile. Results from EUROTRANSFER registry. Am Heart J, 2009; 158: 569-575.

5. Gibson CM, Jennings LK, Murphy SA et al.; INTEGRITI Study Group. Association between platelet receptor occupancy after eptifibatide (Integrilin) therapy and patency, myocardial perfusion, and ST-segment resolution among patients with ST-segment-elevation myocardial infarction: An INTEGRITI (Integrilin and Tenecteplase in Acute Myocardial Infarction) substudy. Circulation, 2004; 110: 679-684.

6. Thiele H, Schindler K, Friedenberger J et al. Intracoronary compared with intravenous bolus abciximab application in patients with ST-elevation myocardial infarction undergoing primary percutaneous coronary intervention: The randomized Leipzig immediate percutaneous coronary intervention abciximab IV versus IC in ST-elevation myocardial infarction trial. Circulation, 2008; 118: 49-57.

7. Bertrand OF, Rodés-Cabau J, Larose E et al. Effects of intracoronary compared to intravenous abciximab administration in patients undergoing transradial percutaneous coronary intervention: A sub-analysis of the EASY trial. Int J Cardiol, 2009; 136: 165-170.

8. Deibele AJ, Jennings LK, Tcheng JE, Neva C, Earhart AD, Gibson CM. Intracoronary eptifibatide bolus administration during percutaneous coronary revascularization for acute coronary syndromes with evaluation of platelet glycoprotein IIb/IIIa receptor occupancy and platelet function: The Intracoronary Eptifibatide (ICE) Trial. Circulation, 2010; 121: 784-791.

9. Bertrand OF, Rodés-Cabau J, Larose E et al.; EArly Discharge after Transradial Stenting of CoronarY Arteries in Acute Myocardial Infarction (EASY-MI) Study Investigators. Intracoronary compared to intravenous Abciximab and high-dose bolus compared to standard dose in patients with ST-segment elevation myocardial infarction undergoing transradial primary percutaneous coronary intervention: A two-by-two factorial placebo-controlled randomized study. Am J Cardiol, 2010; 105: 1520-1527.

10. Gibson CM, Zorkun C, Kunadian V. Intracoronary administration of abciximab in ST-elevation myocardial infarction. Circulation, 2008; 118: 6-8.

11. Møller JE, Søndergaard E, Poulsen SH, Egstrup K. Pseudonormal and restrictive filling patterns predict left ventricular dilation and cardiac death after a first myocardial infarction: A serial color M-mode Doppler echocardiographic study. J Am Coll Cardiol, 2000; 36: 1841-1846.

12. Hillis GS, Møller JE, Pellikka PA et al. Noninvasive estimation of left ventricular filling pressure by $\mathrm{E} / \mathrm{e}$ ' is a powerful predictor of survival after acute myocardial infarction. J Am Coll Cardiol, 2004; 43: 360-367.

13. Schrör K, Weber AA. Comparative pharmacology of GP IIb/IIIa antagonists. J Thromb Thrombolysis, 2003; 15: 71-80.

14. Mehilli J, Kastrati A, Schulz S et al.; Bavarian Reperfusion Alternatives Evaluation-3 (BRAVE-3) Study Investigators. Abciximab in patients with acute ST-segment-elevation myocardial infarction undergoing primary percutaneous coronary intervention after clopidogrel loading: A randomized double-blind trial. Circulation, 2009; 119: 1933-1940.

15. Van't Hof AW, Ten Berg J, Heestermans T et al.Ongoing Tirofiban In Myocardial infarction Evaluation (On-TIME) 2 study group. Prehospital initiation of tirofiban in patients with ST-elevation myocardial infarction undergoing primary angioplasty (On-TIME 2): A multicentre, double-blind, randomised controlled trial Lancet, 2008; 372: 537-546.

16. Stone GW, Witzenbichler B, Guagliumi G et al.; HORIZONS-AMI Trial Investigators. Bivalirudin during primary $\mathrm{PCI}$ in acute myocardial infarction. N Engl J Med, 2008; 358: 2218-2230.

17. Gurm HS, Tamhane U, Meier P, Grossman PM, Chetcuti S, Bates ER. A comparison of abciximab and small molecule glycoprotein IIb/ /IIIa inhibitors in patients undergoing primary percutaneous coronary intervention: A meta-analysis of contemporary randomized controlled trials. Circ Cardiovasc Intervent, 2009; 2: 230-236.

18. De Luca G, Ucci G, Cassetti E, Marino P. Benefits from small molecule administration as compared with abciximab among patients with ST-segment elevation myocardial infarction treated with primary angioplasty: A meta-analysis. J Am Coll Cardiol, 2009; 53: 1668-1673. 
19. Kushner FG, Hand M, Smith SC Jr et al. 2009 focused updates: ACC/AHA guidelines for the management of patients with STelevation myocardial infarction (updating the 2004 guideline and 2007 focused update) and ACC/AHA/SCAI guidelines on percutaneous coronary intervention (updating the 2005 guideline and 2007 focused update) a report of the American College of Cardiology Foundation/American Heart Association Task Force on Practice Guidelines. J Am Coll Cardiol, 2009; 54: 2205-2241.

20. Friedland S, Eisenberg MJ, Shimony A. Meta-analysis of randomized controlled trials of intracoronary versus intravenous administration of glycoprotein IIb/IIIa inhibitors during percutaneous coronary intervention for acute coronary syndrome. Am J Cardiol, 2011; 108: 1244-1251.

21. Navarese EP, Kozinski M, Obonska K et al. Clinical efficacy and safety of intracoronary vs. intravenous abciximab administration in STEMI patients undergoing primary percutaneous coronary intervention: A meta-analysis of randomized trials. Platelets, 2012; 23: 274-281.

22. Thiele H, Wöhrle J, Hambrecht R et al. Intracoronary versus intravenous bolus abciximab during primary percutaneous coronary intervention in patients with acute ST-elevation myocardial infarction: A randomised trial. Lancet, 2012; 379: 923-931.

23. Kubica J, Koziński M, Navarese EP et al. Updated evidence on intracoronary abciximab in ST-elevation myocardial infarction: A systematic review and meta-analysis of randomized clinical trials. Cardiol J, 2012; 19: 230-242.

24. Neumann FJ, Blasini R, Schmitt C et al. Effect of glycoprotein $\mathrm{IIb} / \mathrm{III}$ a receptor blockade on recovery of coronary flow and left ventricular function after the placement of coronary-artery stents in acute myocardial infarction. Circulation, 1998; 98: 2695-2701.

25. Eitel I, Friedenberger J, Fuernau G et al. Intracoronary versus intravenous bolus abciximab application in patients with ST-elevation myocardial infarction undergoing primary percuta- neous coronary intervention: 6-month effects on infarct size and left ventricular function: The randomised Leipzig Immediate PercutaneouS Coronary Intervention Abciximab i.v. versus i.c. in ST-Elevation Myocardial Infarction Trial (LIPSIAbciximab-STEMI). Clin Res Cardiol, 2011; 100: 425-432.

26. Gu YL, Kampinga MA, Wieringa WG et al. Intracoronary versus intravenous administration of abciximab in patients with ST-segment elevation myocardial infarction undergoing primary percutaneous coronary intervention with thrombus aspiration: The Comparison of Intracoronary Versus Intravenous Abciximab Administration During Emergency Reperfusion of ST-Segment Elevation Myocardial Infarction (CICERO) Trial. Circulation, 2010; 122: 2709-2717.

27. Maron BJ, Spirito P, Green KJ, Wesley YE, Bonow RO, Arce J. Noninvasive assessment of left ventricular diastolic function by pulsed Doppler echocardiography in patients with hypertrophic cardiomyopathy. J Am Coll Cardiol, 1987; 10: 733-742.

28. Ommen SR, Nishimura RA, Appleton CP et al. Clinical utility of Doppler echocardiography and tissue Doppler imaging in the estimation of left ventricular filling pressures: A comparative simultaneous Doppler-catheterization study. Circulation, 2000; 102: 1788-1794.

29. Zeymer U, Margenet A, Haude M et al. Eptifibatide as an adjunct to primary $\mathrm{PCI}$ is equally as effective as abciximab with respect to STR. J Am Coll Cardiol, 2010; 56: 463-469.

30. Iversen A, Abildgaard U, Galloe A et al. Intracoronary compared to intravenous bolus abciximab during primary percutaneous coronary intervention in ST-segment elevation myocardial infarction (STEMI) patients reduces 30-day mortality and target vessel revascularization: A randomized trial. J Interv Cardiol, 2011; 24: 105-111.

31. Kubica A, Kozinski M, Navarese EP, Grzesk G, Goch A, Kubica J. Intracoronary versus intravenous abciximab administration in STEMI patients: Overview of current status and open questions. Curr Med Res Opin, 2011; 27: 2133-2144. 